\title{
ORIGINAL ARTICLE \\ Definitions of traumatic conus medullaris and cauda equina syndrome: a systematic literature review
}

\author{
E Brouwers ${ }^{1}, \mathrm{H}$ van de Meent ${ }^{2}, \mathrm{~A} \mathrm{Curt}^{3}, \mathrm{~B}$ Starremans $^{4}, \mathrm{~A} \mathrm{Hosman}^{5}$ and R Bartels ${ }^{1}$
}

\begin{abstract}
Study design: A systematic review.
Objectives: Conus medullaris syndrome (CMS) and cauda equina syndrome (CES) are well-known neurological entities. It is assumed that these syndromes are different regarding neurological and functional prognosis. However, literature concerning spinal trauma is ambiguous about the exact definition of the syndromes.

Methods: A MEDLINE, EMBASE and Cochrane literature search was performed. We included original articles in which clinical descriptions of CMS and/or CES were mentioned in patients following trauma to the thoracolumbar spine.

Results: Out of the 1046 articles, we identified 14 original articles concerning patients with a traumatic CMS and/or CES. Based on this review and anatomical data from cadaveric and radiological studies, CMS and CES could be more precisely defined.

Conclusion: CMS may result from injury of vertebrae Th12-L2, and it involves damage to neural structures from spinal cord segment Th12 to nerve root S5. CES may result from an injury of vertebrae L3-L5, and it involves damage to nerve roots L3-S5. This differentiation between CMS and CES is necessary to examine the hypothesis that CES patients tend to have a better functional outcome.
\end{abstract}

Spinal Cord (2017) 55, 886-890; doi:10.1038/sc.2017.54; published online 23 May 2017

\section{INTRODUCTION}

Traumatic injuries of the thoracolumbar spine can result in conus medullaris syndrome (CMS) or cauda equina syndrome (CES). Fifteen percent of spinal cord injuries (SCI) occur in the thoracolumbar spine. ${ }^{1,2}$ In $\sim 10-38 \%$ of these injuries, the conus medullaris (CM) or cauda equina (CE) is involved; this percentage increases in case of a fracture dislocation. ${ }^{2}$ The clinical symptoms of patients with CMS or CES include the following: low back pain; lower limb weakness; perineum or saddle anesthesia; and bowel and/or bladder dysfunction. Muscle weakness is generally mild, ${ }^{3,4}$ and lower limb weakness associated with CES is predominantly asymmetrical in patients with an incomplete injury. ${ }^{5,6}$ The CMS and CES are assumed to be separate clinical entities, but there is no clear definition of neurological symptoms and level of injury. From a purely anatomical perspective, CMS and CES are separate structures, but clinical distinction between incomplete CMS and CES based upon neurological examination alone is difficult. Cadaveric and magnetic resonance imaging (MRI) studies from 1894 to the present show that the anatomical level of the CM ranges from the lower third of vertebrae Th11 to the upper third of L3, with mean on level L1 (Figure 1). ${ }^{7-17}$

Jefferson was the first author to report compression injury to the $\mathrm{CM}$ and the CE. He described that injury to vertebral level Th12 or L1 might affect the CM. In lower injuries, the CE is affected. ${ }^{18}$ Veraguth was the first author to describe the diagnosis and surgical treatment of a patient with a conus-cauda syndrome. ${ }^{19}$ In 1944, French and Payne described clinical symptoms that were caused by compression of the $\mathrm{CE}$ and named this as 'cauda equina compression syndrome'. ${ }^{20}$ From 1982, CMS and CES were included in the International Standard for Neurological and functional Classification of Spinal Cord Injury (ISNCSCI) by the American Spinal Injury Association (ASIA), but were recently omitted because of the absence of a clear definition. ${ }^{21}$ The CM includes both upper and lower motor neurons, whereas the CE consists solely of lower motor neurons. ${ }^{22}$ It is assumed that the regenerative capacity in lower motor neuron lesions is superior to upper motor neuron lesions, and therefore the functional outcome of CES could be better than the functional outcome of CMS. ${ }^{23}$ For SCI trials, both CMS and CES lesions are often combined as 'conus-cauda lesion', although the outcome between these two syndromes might differ. According to Kirshblum et al., ${ }^{24}$ the level of SCI can be determined by systematically examining the myotomes and dermatomes, whereby injury of the CES may involve only lower motor neurons, and injury to the CM may manifest as a mixed upper and lower motor neuron injury. A clear definition of both syndromes is required to determine functional outcomes, guide treatment decision-making and predict complications. ${ }^{25}$

The aim of this review was to search for the exact definitions of CMS and CES in trauma literature, including the vertebral level of injury, identify neurological symptoms and differentiate between the two syndromes.

${ }^{1}$ Department of Neurosurgery, Radboud University Medical Center, Nijmegen, The Netherlands; ${ }^{2}$ Department of Rehabilitation Medicine, Radboud University Medical Center, Nijmegen, The Netherlands; ${ }^{3}$ Spinal Cord Injury Center, Balgrist University Hospital, Zurich, Switzerland; ${ }^{4}$ Faculty of Medical Sciences, Radboud University, Nijmegen, The Netherlands and ${ }^{5}$ Department of Orthopedics, Radboud University Medical Center, Nijmegen, The Netherlands

Correspondence: E Brouwers, Department of Neurosurgery, Radboud University Medical Center, Geert Grooteplein Zuid 10, 6500 HB Nijmegen, The Netherlands.

E-mail: eveline.brouwers@radboudumc.nl

Received 3 October 2016; revised 31 March 2017; accepted 5 April 2017; published online 23 May 2017 


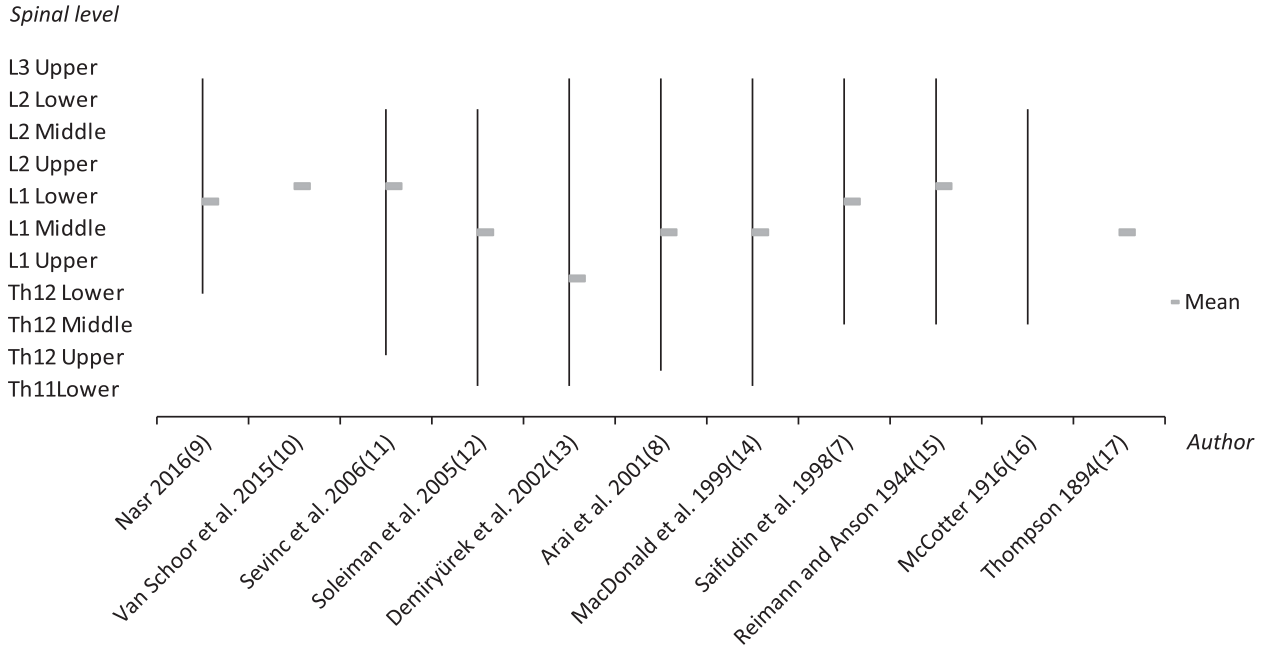

Figure 1 The position of the conus medullaris according to the literature. A full color version of this figure is available at the Spinal Cord journal online.

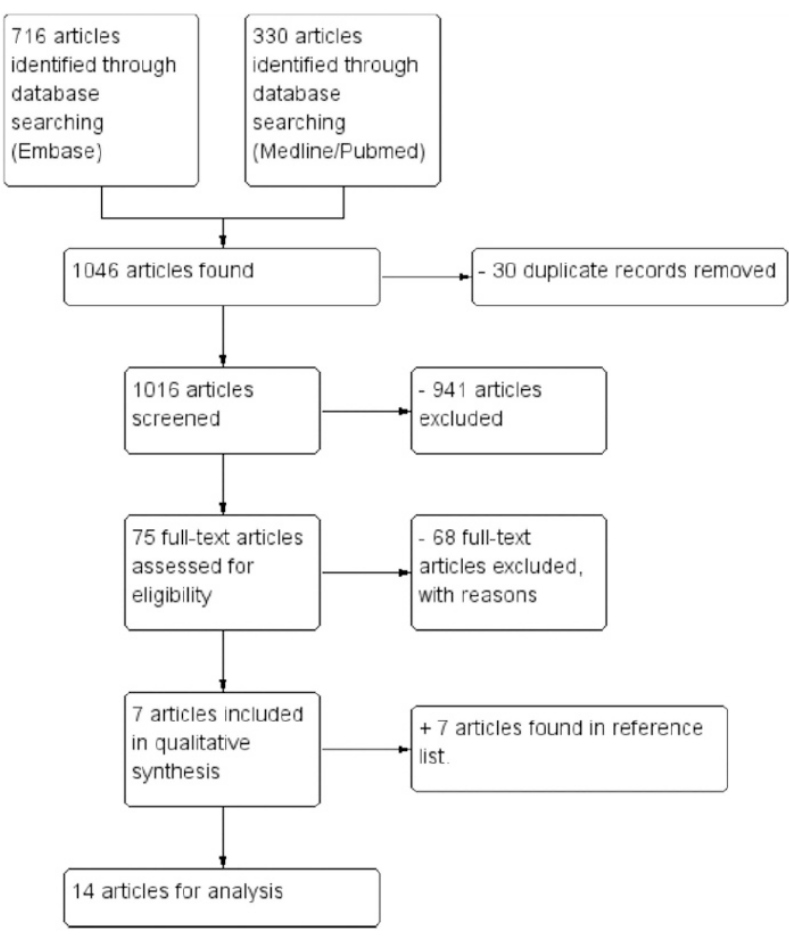

Figure 2 Flow chart of eligible articles.

\section{MATERIALS AND METHODS}

This study was designed and performed according to the Preferred Reporting Items for Systematic reviews and Meta-Analysis (PRISMA) statement. ${ }^{26}$

To answer the question what is the definition of CMS or CES in the trauma literature, a comprehensive electronic database search was performed in Medline (PubMed), Cochrane and Embase by an independent experienced librarian. All papers published up until June 2016 were included. Papers were identified by the following key words: CM, CE, spinal cord syndromes, thoracolumbar junction, thoracolumbar fracture, SCI and trauma. Papers written in English that involved patients with traumatic CMS, CES, traumatic thoracolumbar SCI and included 20 adult patients or more were included. The focus was to find the definition of the CMS and CES; therefore, it was not suitable to criticize the design of the articles. However, only original papers, no reviews, were accepted. Patients with other spinal deficits were excluded.
Because of anatomical diversity, articles concerning children and animals were excluded.

Titles and abstracts were first screened by two investigators in June 2016 (EB and $\mathrm{RB}$ ). Thereafter, the full texts of the eligible articles were screened. Reference lists were screened for missing articles (EB and BS). If the anatomical level or neurological description of the CMS or CES was reported, articles were included.

Because of the narrative design of this study, quality of the included studies and risk on bias were not assessed.

\section{RESULTS}

A total of 1046 articles were initially identified as potentially interesting. Thirty duplicate articles were excluded. After title and abstract screening of 1016 articles, 75 articles were available for full-text analysis. Seven articles reported an anatomical or neurological definition of traumatic CMS or CES. ${ }^{27-33}$ In addition, 7 articles were found in reference lists, ${ }^{4,34-39}$ which provided a total of 14 articles for qualitative analysis (Figure 2). Most articles were retrospective evaluations of single-center studies. The period of published articles spanned from 1982 to 2010 .

\section{Definition of traumatic conus medullaris syndrome}

A compilation of neurological symptoms or vertebral level of the CMS was defined in 14 articles (Table 1). Generally, the vertebral level was used to determine the level of the CM. ${ }^{4,27,29-32,34,35,37,39}$ A fracture (burst fractures and/or distraction dislocation fractures) at level Th12 and L1 was repeatedly associated with CMS. In one article, an affected CMS was observed distally from injury to vertebral level Th10, ${ }^{35}$ and two articles described a CMS from an injured level Th11. ${ }^{32,38}$ The termination level of CM was associated with vertebral levels L1 and L2. Neurological symptoms in patients with CMS were described by McAfee et al., ${ }^{28}$ Clohisy et al..$^{33}$ and Willén et al. ${ }^{36}$ The diagnosis of CMS in the article of McAfee et al. ${ }^{28}$ was based on clinical findings of a neurogenic bladder and loss of third, fourth and fifth sacral nerve root sensation. In the article of Clohisy et al., ${ }^{33}$ the CMS was assessed by clinical evaluation of rectal tone, perianal sensation and the ability to spontaneously void. None of their patients had a complete lesion. Willén et al. ${ }^{36}$ described a CM lesion when there was saddle anesthesia, bladder and/or bowel disturbances or anal problems but no motor impairment in the lower limbs. So, all refer to an acute loss of sensation in the saddle region, bladder and/or bowel dysfunction and 
Table 1 The definitions of CMS found in literature

\begin{tabular}{|c|c|}
\hline Author & Definition of the CM \\
\hline Korovessis et al. ${ }^{32}$ & The lesion was considered to be mixed CMS if the bony level was at Th11-L1. \\
\hline McAfee et al. ${ }^{28}$ & $\begin{array}{l}\text { The determination of } \mathrm{CM} \text { injury was made on the basis of the clinical findings of a neurogenic bladder, and the loss or third, fourth and fifth } \\
\text { sacral nerve root sensation. In patients without a clinically apparent injury of the CM, there was evidence of neural compression with } \\
\text { hyperreflexia, clonus, spasticity and sensory deficit, in addition to compromise of the spinal canal on CT and myelography. }\end{array}$ \\
\hline Kaneda et al. ${ }^{29}$ & Patients with pure CMS had a lesion at vertebral level L1. \\
\hline Transfeldt et al. ${ }^{34}$ & $\begin{array}{l}\text { It appears from this study and others that fractures at T12 and L1 (CM lesions) have a greater potential for neurologic recovery compared to } \\
\mathrm{SCI} \text {, especially of bladder control. }\end{array}$ \\
\hline Rahimi-Movaghar et al. ${ }^{30}$ & In this study, 24 patients presented with CMS. The most frequent level of bony injury was L1, followed by T12. \\
\hline Harrop et al. ${ }^{27}$ & $\begin{array}{l}\text { The lumbar spinal cord or CM contains the anterior horn cells for the distal lumbar and sacral spinal cord segments and is surrounded by nerve } \\
\text { roots for the upper lumbar segments. The lumbar region (Conus) was defined as L1-S5. }\end{array}$ \\
\hline Kim et al..$^{31}$ & There were 48 fractures in the $\mathrm{CM}$ area $(\mathrm{L} 1)$ \\
\hline Clohisy et al. ${ }^{33}$ & $\begin{array}{l}\text { CM function was assessed by clinical evaluation of rectal tone, perianal sensation and the ability to spontaneously void. Out of } 20 \text { patients, } \\
15 \text { patients with a fracture at T12 or L1 had a CMS. All patients with a fracture at L1 had a CMS; however, not every patient with a T12 } \\
\text { fracture had CMS. }\end{array}$ \\
\hline Jelsma et al. ${ }^{35}$ & In this study, 50 patients had thoracolumbar fractures and complete or incomplete CMS or CES. Patients had fractures from Th10 to L2. \\
\hline Blumenkopf and Juneau ${ }^{37}$ & The CM was observed at the th12 level in 8 patients, the L1 level in 10 patients and the L2 level in 2 patients. \\
\hline Podnar 4 & Patients with pathology at the T12 or L1 levels were assumed to have CM lesions. \\
\hline Gertsbein et al. ${ }^{38}$ & The lesion was considered to be mixed CMS and CES if the fracture was located at Th11-L1. \\
\hline Bradford and McBride 39 & The conus portion of the spinal cord is usually found between $L 1$ and $L 2$. \\
\hline Willén et al.36 & $\begin{array}{l}\text { A conus lesion was identified, when there was saddle anesthesia, bladder and/or bowel disturbances or sexual problems, but no motor } \\
\text { impairment in the lower limbs. }\end{array}$ \\
\hline
\end{tabular}

Abbreviations: CES, cauda equina syndrome; CMS, conus medullaris syndrome; CT, computed tomography; SCI, spinal cord injury.

\section{Table 2 The definitions of CES found in literature}

\begin{tabular}{ll}
\hline Author & Definition of CE \\
\hline Korovessis et al. ${ }^{32}$ & The lesion was considered to be CES, if bony level was L2 or below. \\
Kaneda et al. & Patients with pure CES presented a lesion at vertebral level L2 and below. \\
Kim et al. & There were 59 fractures in the level of CE (L2, L3 and L4). \\
Podnar $^{4}$ & Patients with pathology below the L1-L2 intervertebral level were assumed to have CE lesions. \\
Gertsbein et al. $^{38}$ & The lesion was considered to be pure CES, if the bony level was L2 or below. \\
Bradford and McBride ${ }^{39}$ & The CE was below L2. \\
Willén et al. ${ }^{36}$ & In patients with signs of nerve root lesions without bladder or bowel disturbances, saddle anesthesia or sexual problems, the term used was \\
& CE lesion.
\end{tabular}

Abbreviation: CES, cauda equina syndrome.

Definition of CES was not available in the articles of McAfee et al., ${ }^{28}$ Transfeldt et al.., ${ }^{34}$ Rahimi-Movaghar et al., ${ }^{30}$ Harrop et al., ${ }^{27}$ Clohisy et al., ${ }^{33}$ Jelsma et al., ${ }^{35}$ Blumenkopf and Juneau. ${ }^{37}$

sexual dysfunction after trauma, without muscle weakness in lower extremities.

\section{Definition of traumatic cauda equina syndrome}

Seven articles were identified that described traumatic CES (Table 2). Most studies identify vertebral level (L2) as the cranial border of the CE. ${ }^{29,31,32,38,39}$ Podnar described pathology caudal from level L1 to L2 to result in CES. ${ }^{4}$ Only Willén et al. ${ }^{36}$ described the neurological symptoms of a traumatic CES - signs of nerve root lesion without saddle anesthesia, bladder and/or bowel dysfunction or sexual dysfunction.

According to Podnar, the definition of a traumatic CMS and CES is based on the following: (1) a history of a causal event followed by lower limb weakness, bladder and/or bowel or sexual dysfunction; (2) clinical examination-reduced sphincter contraction, sacral sensory loss or, in men, a diminished penilocavernosus reflex; (3) signs of denervation on electromyographic; (4) decreased sacral reflexes; and (5) radiological findings of lumbosacral pathology. In his article, Podnar could not differentiate the CMS and CES based on neurological outcomes because in 20 patients with a CMS, additional root lesion could not be expelled. Patients with a lesion at Th12-L1 were assumed to have a CMS; pathology at L1-L2 intervertebral level and below was associated with a CES. ${ }^{4}$

Mixed CMS/CES cases were described with vertebral lesions between Th10 and L1, whereby no differentiation was made based on neurological function (Table 3 ). $4,27,29,32,36,38$

\section{DISCUSSION}

The articles included in this review indicate that the definition of both traumatic CMS and CES in the acute phase is based on radiological findings. A distinction can be derived by level of injury whereby trauma to vertebral level Th12-L2 will likely cause a CMS, whereas damage to vertebral level L3 and below will cause a CES. However, in the traumatology literature a distinction is not made on neurological examination in the acute setting. Patients with CMS tend to have symmetric sensory-motor deficits, whereas patients with CES tend to have more asymmetric sensory-motor deficits. ${ }^{4,22,28,33,36}$ These findings correspond with previous reviews by Wagner and Jagoda, ${ }^{3}$ Orendacova et al. ${ }^{5}$ and Fraser et al. ${ }^{6}$ Our review was established from trauma literature whereby the diagnosis was based on motor and sensor examination and radiology in the acute phase. Bladder function 
Table 3 Neurological symptoms of CMS and CES

\begin{tabular}{|c|c|c|c|}
\hline Symptoms & $C M$ & $C E$ & References \\
\hline Low back pain, accompanied by pain radiating into one or both legs. & Yes & Yes & $4-6,35$ \\
\hline Muscle weakness in lower extremities & Predominantly symmetrical & Often asymmetrical & $3-6,35$ \\
\hline Bladder and rectal sphincter dysfunction & Yes & Yes & $4-6,32-35$ \\
\hline
\end{tabular}

Abbreviations: CES, cauda equina syndrome; CMS, conus medullaris syndrome.

Table 4 Proposal for the definition of the CMS and the CES

\begin{tabular}{lccc}
\hline & Vertebral level of injury & Neurological level of injury & ISNCSI level of injury \\
\hline CMS & T12-L2 & T12-S5 & T11 \\
CES & L3-L5 & L3-S5 & L2 \\
\hline
\end{tabular}

Abbreviations: CES, cauda equina syndrome; CMS, conus medullaris syndrome; ISNCSI, International Standard for Neurological and functional Classification of Spinal Cord Injury.

is an important clinical sign to distinguish between CMS and CES. However, we did not find any literature dealing with this topic in the acute setting. Most of these patients already have an indwelling urinary catheter, and further investigations on this topic are not warranted in the emergent situation. We did not find data of additional physical or neurophysical examination directly after trauma to specify the diagnosis.

The anatomical level of the CM was described by Thomson in $1894 .{ }^{17}$ In his report of collective investigations of 198 cadavers, the average termination of the CM was situated at vertebral level L1. More recent cadaveric and MRI studies have shown the same results, indicating that the tip of the CM is situated at the (middle/lower third of) vertebral level L1 (Table 1). There is a sex difference in the mean termination level of the CM, whereas the spinal cord in females tends to be longer compared to that in males-L1-L2 vs Th12-L1 intervertebral space. ${ }^{7,11,13,14}$ However, only in the study of Demiryürek et al. ${ }^{13}$ is this difference significant. In newborns and infants up to the age of 12, the mean termination level of the tip of the $\mathrm{CM}$ is at L2-L3. The mean termination level of the CM in adolescents and early adults (age 13-29) is at L1-L2. ${ }^{10}$ Anatomy studies support the assumption, derived from the trauma literature in this review, that the tip of the CM is situated between Th12 and L2. It should be noted that the individuals in our included articles, as well as those in the anatomy studies, did not have any spinal deformities.

The minimal number of patients who were reported in a study to be included might be considered a limitation. However, while designing the study, we assumed that the quality of the results of studies reporting on very few patients can be questioned. Furthermore, we are confident that inclusion of a small case series will not alter the conclusions of our study. Therefore, we did not introduce them after the results were obtained. Another flaw could be the focus on traumatic lesions. We were interested in traumatic CMS and CES because we assumed that there is no differentiation between these syndromes in the acute phase. Therefore, we have limited our search to traumatic CMS and CES, despite the fact that more causes are known for these syndromes-for example, tumor and hernia nucleus pulposus. Moreover, a major difference between a traumatic SCI and non-traumatic cases is the acute moment of injury to the spine. Because of acute compression, secondary ischemia, hemorrhage and/or edema may occur. Therefore, its course can be different from non-traumatic CMS/CES. The articles were retrospective single-center studies, presenting neurological outcomes after surgery. We used these articles to investigate which definition of the CMS and the CES was

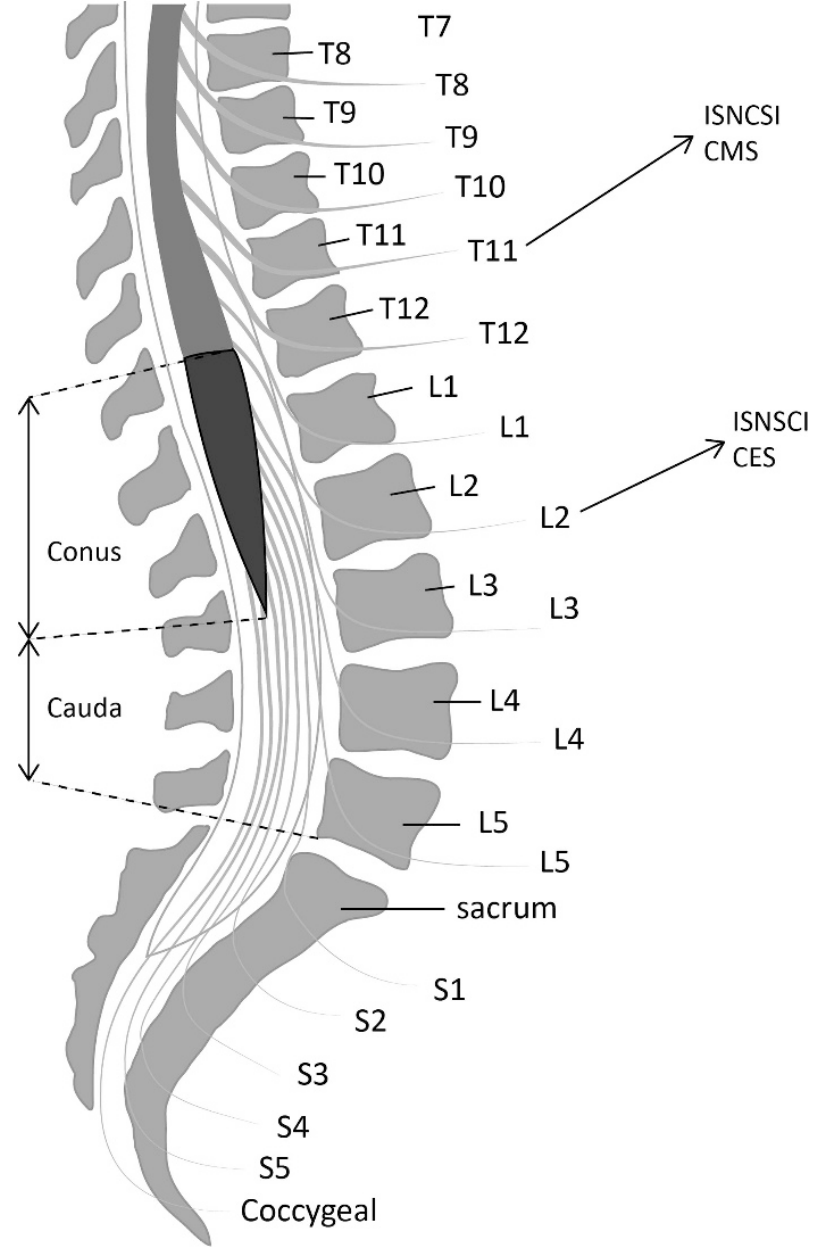

Figure 3 Schematic presentation of the conus medullaris and cauda equina. A full color version of this figure is available at the Spinal Cord journal online.

used. The low quality of the studies is a matter of concern. However, as CMS and CES were only descriptions of the subject of research and not of an outcome itself, we are convinced that these were reliable and did not affect our results and conclusions.

The strength of this study arises from our search for combined neurological, radiological and anatomical studies to find differences between the CMS and the CES. Until now, the diagnosis of CMS and the CES was based on non-specific neurological symptoms (low back pain, lower limb weakness, perineum or saddle anesthesia and bowel and/or bladder dysfunction) in cases with injury to the lower thoracic or lumbar spinal cord. X-ray, computerized axial tomography and MRI scans seem to be the deciding factor for the diagnosis. However, besides the initial level of injury, it is important to recognize the syndrome at an early stage to predict neurological recovery, ${ }^{40}$ 
especially as the prognosis of the CES might be superior compared to CMS. ${ }^{23}$ From the standpoint of decision-making and informing the patient and family, it is important to minimize ambiguity about these syndromes. To add the ISNCSCI gradation to our results from the literature (Table 4), a clear cutoff point for the border between CMS and CES could be derived. This addition is necessary in future research to examine the hypothesis that CES patients tend to have a better functional outcome.

\section{CONCLUSION}

A CMS may result from a trauma to vertebra T12-L2 in combination with neurological impairment in dermatomyotomes Th12-S5. The most cranial ISNCSCI level for CMS is Th11. A trauma to vertebra L3-L5 with neurological impairment of the nerve roots is always a CES in patients without pre-existing spinal deformities. The most cranial ISNCSCI level for CES is L2. MRI is the gold standard for the discrimination between CMS and CES. These findings are necessary to distinguish the CMS from the CES, whereby the differences in functional outcome between these patient groups can be investigated Figure 3 .

\section{DATA ARCHIVING}

There were no data to deposit.

\section{CONFLICT OF INTEREST}

The authors declare no conflict of interest.

\section{ACKNOWLEDGEMENTS}

This paper was written without sources of support.

1 Sekhon LH, Fehlings MG. Epidemiology, demographics, and pathophysiology of acute spinal cord injury. Spine 2001; 26: S2-S12.

2 Looby S, Flanders A. Spine trauma. Radiol Clin North Am 2011; 49: 129-163.

3 Wagner R, Jagoda A. Spinal cord syndromes. Emerg Med Clin North Am 1997; 15: 699-711.

4 Podnar S. Epidemiology of cauda equina and conus medullaris lesions. Muscle Nerve 2007; 35: 529-531.

5 Orendacova J, Cizkova D, Kafka J, Lukacova N, Marsala M, Sulla I et al. Cauda equina syndrome. Prog Neurobiol 2001; 64: 613-637.

6 Fraser S, Roberts L, Murphy E. Cauda equina syndrome: a literature review of its definition and clinical presentation. Arch Phys Med Rehabil 2009; 90: 1964-1968.

7 Saifuddin A, Burnett SJ, White J. The variation of position of the conus medullaris in an adult population. A magnetic resonance imaging study. Spine (Phila Pa 1976) 1998; 23: $1452-1456$.

8 Arai $\mathrm{Y}$, Shitoto $\mathrm{K}$, Takahashi M, Kurosawa $\mathrm{H}$. Magnetic resonance imaging observation of the conus medullaris. Bull Hosp Jt Dis 2001; 60: 10-12.

9 Nasr AY. Clinical relevance of conus medullaris and dural sac termination level with special reference to sacral hiatus apex: anatomical and MRI radiologic study. Anat Sci Int (e-pub ahead of print 19 April 2016; doi: 10.1007/s12565-016-0343-0).

10 Van Schoor AN, Bosman MC, Bosenberg AT. Descriptive study of the differences in the level of the conus medullaris in four different age groups. Clin Anat 2015; 28: 638-644.

11 Sevinc O, Is M, Barut C, Eryoruk N, Kiran S, Arifoglu Y. MRI determination of conus medullaris level in an adult population in Turkey. Neuroradiol J 2006; 19: 375-378.

12 Soleiman J, Demaerel P, Rocher S, Maes F, Marchal G. Magnetic resonance imaging study of the level of termination of the conus medullaris and the thecal sac: influence of age and gender. Spine (Phila Pa 1976) 2005; 30: 1875-1880.
13 Demiryurek D, Aydingoz U, Aksit MD, Yener N, Geyik PO. MR imaging determination of the normal level of conus medullaris. Clin Imaging 2002; 26: 375-377.

14 Macdonald A, Chatrath P, Spector T, Ellis H. Level of termination of the spinal cord and the dural sac: a magnetic resonance study. Clin Anat 1999; 12: 149-152.

15 Reiman AF, Anson BJ. Vertebral level of termination of the spinal cord with report of a case of a sacral cord. Anat Rec 1944; 88: 127-138.

16 McCotter RE. Regarding the length and extent of the human medulla spinalis. Anat Rec 1916; 10: 559-564.

17 Thomson A. Fifth Annual Report of the Committee of Collective Investigation of the Anatomical Society of Great Britain and Ireland for the Year 1893-94. J Anat Physiol 1894; 29: 35-60.

18 Jefferson G. Discussion on spinal Injuries. Proc R Soc Med 1928; 21: 625-648.

19 Stienen MN, Surbeck W, Trohler U, Hildebrandt G. Little-known Swiss contributions to the description, diagnosis, and surgery of lumbar disc disease before the Mixter and Barr era. J Neurosurg Spine 2013; 19: 767-773.

20 French JD, Payne JT. Cauda equina compression syndrome with herniated nucleus pulposus: a report of eight cases. Ann Surg 1944; 120: 73-87.

21 Kirshblum S, Waring W 3rd. Updates for the International Standards for Neurological Classification of Spinal Cord Injury. Phys Med Rehabil Clin N Am 2014; 25: 505-517.

22 Radcliff KE, Kepler CK, Delasotta LA, Rihn JA, Harrop JS, Hilibrand AS et al. Current management review of thoracolumbar cord syndromes. Spine J 2011; 11: 884-892.

23 Tator $\mathrm{CH}$. Biology of neurological recovery and functional restoration after spinal cord injury. Neurosurgery 1998; 42: 696-707; discussion 707-708.

24 Kirshblum SC, Burns SP, Biering-Sorensen F, Donovan W, Graves DE, Jha A et al. International standards for neurological classification of spinal cord injury (revised 2011). J Spinal Cord Med 2011; 34: 535-546.

25 van Middendorp JJ, Audige L, Hanson B, Chapman JR, Hosman AJ. What should an ideal spinal injury classification system consist of? A methodological review and conceptual proposal for future classifications. Eur Spine J 2010; 19: 1238-1249.

26 Moher D, Liberati A, Tetzlaff J, Altman DG. Preferred reporting items for systematic reviews and meta-analysis: the PRISMA statement. PLos Med 2009; 6: e1000097.

27 Harrop JS, Naroji S, Maltenfort MG, Ratliff JK, Tjoumakaris SI, Frank B et al. Neurologic improvement after thoracic, thoracolumbar, and lumbar spinal cord (conus medullaris) injuries. Spine (Phila Pa 1976) 2011; 36: 21-25.

28 McAfee PC, Bohlman HH, Yuan HA. Anterior decompression of traumatic thoracolumbar fractures with incomplete neurological deficit using a retroperitoneal approach. J Bone Joint Surg Am 1985; 67: 89-104.

29 Kaneda K, Abumi K, Fujiya M. Burst fractures with neurologic deficits of the thoracolumbar-lumbar spine. Results of anterior decompression and stabilization with anterior instrumentation. Spine 1984; 9: 788-795.

30 Rahimi-Movaghar V, Vaccaro AR, Mohammadi M. Efficacy of surgical decompression in regard to motor recovery in the setting of conus medullaris injury. J Spinal Cord Med 2006; 29: 32-38.

$31 \mathrm{Kim} \mathrm{NH}$, Lee HM, Chun IM. Neurologic injury and recovery in patients with burst fracture of the thoracolumbar spine. Spine 1999; 24: 290-293; discussion 294.

32 Korovessis P, Piperos G, Sidiropoulos P, Karagiannis A, Dimas T. Spinal canal restoration by posterior distraction or anterior decompression in thoracolumbar spinal fractures and its influence on neurological outcome. Eur Spine J 1994; 3: 318-324.

33 Clohisy JC, Akbarnia BA, Bucholz RD, Burkus JK, Backer RJ. Neurologic recovery associated with anterior decompression of spine fractures at the thoracolumbar junction (T12-L1). Spine 1992; 17: S325-S330.

34 Transfeldt EE, White D, Bradford DS, Roche B. Delayed anterior decompression in patients with spinal cord and cauda equina injuries of the thoracolumbar spine. Spine 1990; 15: 953-957.

35 Jelsma RK, Kirsch PT, Jelsma LF, Ramsey WC, Rice JF. Surgical treatment of thoracolumbar fractures. Surg Neurol 1982; 18: 156-166.

36 Willén J, Anderson J, Toomoka K, Singer K. The natural history of burst fractures at the thoracolumbar junction. J Spinal Disord 1990; 3: 39-46.

37 Blumenkopf B, Juneau PA 3rd. Magnetic resonance imaging (MRI) of thoracolumbar fractures. J Spinal Disord 1988; 1: 144-150.

38 Gertzbein SD, Court-Brown CM, Marks P, Martin C, Fazl M, Schwartz M et al. The neurological outcome following surgery for spinal fractures. Spine 1988; 13: 641-644.

39 Bradford DS, McBride GG. Surgical management of thoracolumbar spine fractures with incomplete neurologic deficits. Clin Orthop Relat Res 1987; 218: 201-216.

40 Kirshblum SC, O'Connor KC. Predicting neurologic recovery in traumatic cervical spinal cord injury. Arch Phys Med Rehabil 1998; 79: 1456-1466. 